\title{
openheart FLOWER-MI and the root of the problem with non-culprit revascularisation
}

\author{
Matthew E Li Kam Wa (1) ,1,2 Kalpa De Silva, ${ }^{1,2}$ Carlos Collet, ${ }^{3}$ Divaka Perera ${ }^{1,2}$
}

To cite: Li Kam Wa ME, De Silva K, Collet C, et al. FLOWER-Ml and the root of the problem with non-culprit revascularisation. Open Heart 2021;8:e001763. doi:10.1136/ openhrt-2021-001763

Accepted 12 October 2021

\section{Check for updates}

(c) Author(s) (or their employer(s)) 2021. Re-use permitted under CC BY-NC. No commercial re-use. See rights and permissions. Published by BMJ.

${ }^{1}$ Cardiovascular Division, Guy's and St Thomas' NHS Foundation Trust, London, UK

${ }^{2}$ British Heart Foundation Centre of Excellence and National Institute for Health Research Biomedical Research Centre, King's College London, London, UK

${ }^{3}$ Cardiovascular Center, Onze Lieve Vrouw Ziekenhuis, Aalst, Belgium

\section{Correspondence to} Dr Matthew E Li Kam Wa; mlikamwa@nhs.net

\section{ABSTRACT}

How do we reduce cardiac death and myocardial infarction by percutaneous coronary intervention (PCl) in coronary heart disease? Although the interventional community continues to grapple with this question in stable angina, the benefits of PCl for non-culprit lesions found at STelevation myocardial infarction are established. Is it then wishful thinking that an index developed in stable coronary disease, for identifying lesions capable of causing ischaemia will show an incremental benefit over angiographically guided non-culprit PCI? This is the question posed by the recently published FLOW Evaluation to Guide Revascularization in Multi-vessel ST-elevation Myocardial Infarction (FLOWER-MI) trial. We examine the trial design and results; ask if there is any relationship between the baseline physiological significance of a non-culprit lesion and vulnerability to future myocardial infarction; and consider if more sophisticated methods can help guide or defer non-culprit revascularisation.

\section{INTRODUCTION}

Randomised trials involving more than 6300 patients over the last decade all agree that revascularisation of non-culprit lesions at ST-elevation myocardial infarction (STEMI) is superior to medical therapy alone. ${ }^{1}$ However, it is uncertain if fractional flow reserve (FFR) is beneficial in deciding which non-culprit lesions to revascularise. FLOW Evaluation to Guide Revascularization in Multi-vessel ST-elevation Myocardial Infarction (FLOWER-MI) was an investigator-initiated, multicentre trial conducted over 2 years in France, that randomised 1171 patients following STEMI with an angiographic stenosis of $\geq 50 \%$ in at least one non-culprit artery to FFR or angiographic guided percutaneous coronary intervention (PCI). ${ }^{2}$ All patients underwent nonculprit assessment prior to discharge. There was no difference in the primary endpoint which was a composite of all cause death, non-fatal myocardial infarction (MI), or unplanned hospitalisation leading to urgent revascularisation at 1 year (HR 1.32 for FFR vs angiography; $95 \%$ CI 0.78 to 2.23 ). These results generated debate at the American College of Cardiology Scientific Sessions and were recently published in full in the New
England Journal of Medicine. Now that the dust has settled, what can we take from FLOWER-MI beyond the headlines?

\section{FLOWER-MI: a closer look}

The trial was powered to detect a $5.5 \%$ absolute reduction in the incidence of the primary endpoint and all prespecified clinical outcomes were not significantly different between FFR and angiography groups at 1 year. Why did FFR not perform as well as hoped? It is worth noting that 7 out of $9(78 \%)$ deaths in the FFR group were non-cardiac, vs only 3 of $10(30 \%)$ in the angiography group. Non-fatal MI was also numerically higher in the FFR group and despite less PCI being performed, the periprocedural MI (type 4a) rate was three times higher. The $0.3 \%$ periprocedural MI rate within the angiography arm, however, is almost certainly underreported, given the challenges in diagnosing periprocedural MI around the time of STEMI, when troponin levels are already elevated and/or still rising. Compliance to troponin collection is not reported, nor the number of troponin samples taken per patient. The overall MI rate within deferred patients within the FFR arm was high at $5.6 \%$, with divergence in the Kaplan-Meier curves at 6 months from the index procedure. However, we also do not know which events occurred in deferred lesions and ultimately attempts to draw conclusions are limited by the low numbers of events, which were only onethird of what the authors anticipated.

To understand this, we should examine the trial design in more detail. At first glance, the randomisation of FLOWER-MI bears some resemblance to the stable coronary disease cohort of FAME (Fractional Flow Reserve Versus Angiography for Multivessel Evaluation). ${ }^{3}$ Unfortunately, there was no blinded assessment of FFR within the angiography guided arm and it is uncertain why the pre-PCI FFR result was not available for $154(16 \%)$ of lesions. Why is this important? Any study depends on a difference in exposure between the two arms to show a treatment effect and discordance between FFR and angiographic severity is most pronounced in the intermediate $(<70 \%)$ range. Considering 


\begin{tabular}{|c|c|c|c|c|c|}
\hline & \multicolumn{2}{|c|}{ FLOWER-MI } & \multirow[t]{2}{*}{ COMPLETE } & \multirow[t]{2}{*}{ Compare-Acute } & \multirow[t]{2}{*}{ DANAMI-3-PRIMULTI } \\
\hline & Angio & FFR & & & \\
\hline Dates & \multicolumn{2}{|c|}{ 2016-2018 } & 2011-2015 & 2011-2015 & 2011-2014 \\
\hline No of patients & \multicolumn{2}{|l|}{1171} & 4041 & 885 & 627 \\
\hline Median follow-up (mths) & \multicolumn{2}{|l|}{12} & 36 & 12 & 27 \\
\hline Design & \multicolumn{2}{|c|}{ Angio (>50\%) vs FFR } & Angio $(\geq 70 \%)^{*}$ vs OMT & FFR vs OMT & FFR vs OMT \\
\hline $\mathrm{FFR} \geq 0.80$ & \multicolumn{2}{|l|}{$44 \%$} & - & $46 \%$ & $31 \%$ \\
\hline \multirow[t]{2}{*}{$\begin{array}{l}\text { Composite primary outcome } \\
\text { (no/\%) }\end{array}$} & \multicolumn{2}{|c|}{$\begin{array}{l}\text { Death, non-fatal MI, unplanned } \\
\text { admission and urgent revasc }\end{array}$} & $\begin{array}{l}\text { CV death, MI, } \\
\text { ischaemia-driven } \\
\text { revasc }\end{array}$ & $\begin{array}{l}\text { Death, non-fatal MI, any } \\
\text { revasc, CVA/TIA }\end{array}$ & $\begin{array}{l}\text { Death, non-fatal MI, ischaemia- } \\
\text { driven revasc }\end{array}$ \\
\hline & $24(4.2)$ & $32(5.5)$ & $179(8.9)$ & $23(7.8)$ & $40(13)$ \\
\hline \multicolumn{6}{|l|}{$\begin{array}{l}\text { Event rate with } \\
\text { revascularisation (\%)† }\end{array}$} \\
\hline Death & 1.7 & 1.5 & 1.6 & 1.4 & 4.7 \\
\hline CV death & NR & & 1.0 & 1.0 & 1.6 \\
\hline $\mathrm{Ml}$ & 1.7 & 3.1 & 1.9 & 2.4 & 4.7 \\
\hline
\end{tabular}

${ }^{*}$ Only a minority of patients with moderate stenoses (50\%-69\%) that would have mandated FFR measurement were enrolled in COMPLETE. tEvent rates for COMPLETE shown as \% per person-year as reported by the authors, for comparison with the 12-month follow-up of FLOWER-MI.

COMPLETE, Complete versus Culprit-Only Revascularisation Strategies to Treat Multivessel Disease after Early PCI for STEMI; CV, cardiovascular; CVA, cerebrovascular accident; DANAMI-3-PRIMULTI, Third Danish Study of Optimal Acute Treatment of Patients with STEMI: Primary PCI in Multivessel Disease; FFR, fractional flow reserve; FLOWER-MI, FLOW Evaluation to Guide Revascularization in Multi-vessel ST-elevation Myocardial Infarction; MI, myocardial infarction; NR, not formally reported; OMT, optimal medical therapy; PCI, percutaenous coronary intervention; STEMI, ST-elevation myocardial infarction; TIA, transient ischaemic attack.

the inclusion of severe stenoses in this study and that intermediate disease comprised less than half of all lesions, FFR changed the treatment strategy in only $40 \%$ of lesions. This is in keeping with both DANAMI-3-PRIMULTI (Third Danish Study of Optimal Acute Treatment of Patients with STEMI: Primary PCI in Multivessel Disease) and Compare-Acute cohorts ( $31 \%$ and $46 \%$, respectively). At the patient level, this translated to only one-third (198 of 586 patients) not having non-culprit PCI. Relying on this minority to show a statistically significant difference in outcomes was always going to be a challenge. Although this trial was designed prior to the results of COMPLETE (Complete Versus Culprit-Only Revascularization Strategies to Treat Multivessel Disease After Early PCI for STEMI), ${ }^{4}$ the estimated event rate of $15 \%$ in the angiography guided group still seems generous given the 1-year primary outcome rate of approximately $5 \%$ in PRAMI (Preventive Angioplasty in Acute Myocardial Infarction) and $10 \%$ in CvLPRiT (complete versus lesion-only primary PCI). FLOWER-MI now joins the list of non-culprit revascularisation trials, but with a comparatively low primary outcome rate of $4.2 \%$ in the angiographic arm vs $5.5 \%$ in the FFR arm (table 1).

Why the marked differences in event rates between these trials, and why do these rates differ from real-world registries? The baseline characteristics of the randomised non-culprit trials are largely comparable, although the complexity of non-culprit disease is not uniformly reported. A lower-risk population may be indicated by the $23 \%$ of patients in FLOWER-MI with 3 vessel disease vs the $36 \%$ in PRAMI.
There are also differences such as the rates of glycoprotein $\mathrm{IIb} / \mathrm{III} a$ and P2Y12 inhibitor use, although this is reflective of optimal therapy at the time of each study. However, a more significant contributor is likely to be the open label design of these trials.

\section{Open label trials and caveats}

In the era of primary PCI and modern medical therapy, it is increasingly difficult to show benefits in objective, 'hard' endpoints without large, and therefore, expensive trials. Open label trials conflated with 'soft' outcomes such as hospitalisation and urgent revascularisation renders them susceptible to bias, but also makes it difficult to distinguish the true symptomatic and prognostic effects of PCI. In this regard, a strength of COMPLETE was powering for a coprimary endpoint with the objective outcomes of cardiovascular death and MI. We also caution against extrapolating results from any STEMI trial to the non-ST-elevation MI population who have more comorbidities, more complex coronary disease and the culprit lesion is frequently ambiguous or determined by using the angiogram alone.

\section{Impact on clinical practice}

\section{Could less be more?}

Given the similar risk of MI and revascularisation in previous FFR and angiographic guided trials versus medical therapy (table 1), FLOWER-MI certainly addresses clinically important questions. Is there a middle ground where the benefits of 'complete' (or 
COMPLETE) revascularisation can be gained, without stenting every angiographic lesion? Unfortunately, given the lack of statistical difference in its outcomes, the answers remain unclear. We believe it continues to remain reasonable to offer either an angiographic or FFR guided strategy. Based on expected discordance rates, ${ }^{5}$ an FFR strategy versus the (almost entirely) angiographic strategy of COMPLETE for $>70 \%$ stenoses $^{4}$ would be expected to still revascularise $85 \%$ of lesions.

If this is the case, should we quibble over a $15 \%$ reduction in lesion revascularisation? In our view, it is important to remember that PCI should not be regarded as a benign procedure. Aside from the periprocedural risk, underreported in FLOWER-MI, at a mean age of 62 years these patients would likely live another 20 years, accruing risk of very-late stent-related events year-on-year. ${ }^{6}$ This may be an acceptable trade off on an individualised basis, but not if stent implantation has no prospect of improving their prognosis in the first place. We believe that FLOWER-MI provides a potentially simple and important message, FFR can safely defer $1 / 3$ of non-culprit lesions noted at the time of STEMI.

\section{Is physiological assessment valid at the time of STEMI?}

FLOWER-MI aside, we should consider the validity of physiological coronary assessment in acute coronary syndromes (ACS). We know the measurement of intracoronary pressure as a pragmatic surrogate for flow reduces urgent revascularisation, spontaneous $\mathrm{MI}$ and is cost effective in those with stable coronary artery disease planned for PCI. ${ }^{7}$ Physiological assessment to improve symptoms has a clear rationale here: if ischaemia is required to cause angina, intervention in the absence of flow limitation exposes the patient to the risk of a procedure, while also being unhelpful for their angina.

Due to changes in microvascular physiology at the time of STEMI, concern exists whether indices such as FFR or iFR, (both developed and validated within the stable coronary artery disease population) accurately reflect functional significance. This is evaluated in detail by a recent review, ${ }^{1}$ but importantly, flow as well as hyperaemic and resting pressure indices can be overestimated or underestimated during the index admission. These changes could have significant impact around the dichotomous treatment thresholds established for stable angina. Although studies are small, conflicting, and the long-term clinical implications are not characterised - up to one in five lesions deferred by FFR, and one in three lesions treated by iFR could have a different treatment if reassessed 30 days later.

\section{Why do patients have adverse events after culprit revascularisation?}

If physiological indices are prone to inaccuracy after STEMI, why not simply pursue angiographic-guided revascularisation? Serial angiography and pathological studies certainly support that culprit lesions at the time of STEMI are severely stenotic. ${ }^{8}$ However, to adopt this approach would be to disregard compelling evidence about the origin of adverse events after STEMI.

FLOWER-MI does not allow us to attribute events to culprit or non-culprit lesions (deferred by FFR or otherwise) but vessel level outcomes were reported in a sub study of Compare-Acute, which had the benefit of measuring non-culprit FFR in both arms. ${ }^{9}$ In this study, non-culprit, angiographically insignificant vessels (that did not warrant FFR measurement in the first place) were responsible for two thirds of the spontaneous MIs within the follow-up period.

For a systematic multimodality evaluation and natural history of non-culprit lesions, we should turn our attention to the contemporary PROSPECT II study. ${ }^{10}$ After ACS (22\% STEMI), 898 patients underwent FFR guided revascularisation of all $>40 \%$ nonculprit lesions, followed by three-vessel intravascular ultrasound (IVUS) and follow-up for 4 years. Although we must be cautious in extrapolating data from a combined ACS cohort, of the major adverse cardiac events, two-thirds were attributable to non-culprit lesions. Of the non-culprit lesions which underwent intracoronary imaging characterisation at baseline, the mean diameter stenosis was only $47 \%$. Clearly, a strategy of angiographic-guided revascularisation for $>50 \%-70 \%$ stenoses is liable to miss a significant number of potentially clinically relevant lesions.

Conversely, not all severely stenotic lesions at baseline are destined to cause a future adverse event. This broad heterogeneity in coronary plaque vulnerability, haemodynamic stresses and the risk profile of patients with coronary artery disease explains why some lesions lead to MI and others do not. Plaques that are more likely to rupture or erode are termed 'vulnerable' and have distinct features that can be identified by dedicated imaging techniques. When combined with patient level factors such as diabetes, lifestyle factors or the pro-inflammatory state of STEMI, these unstable plaques can lead to acute coronary syndromes (ACS).

\section{Vulnerable plaque and vulnerable patient assessment}

Intracoronary imaging has focused on identifying the pathological features associated with thrombotic occlusions: virtual histology IVUS (VH-IVUS: thin cap fibroatheroma, plaque burden and minimum lumen area), nearinfrared spectroscopy (NIRS: lipid rich plaque) and optical coherence tomography (OCT: lipid arc, cap thickness and macrophages) which seem to be more prevalent in angiographically severe lesions. ${ }^{11}$ However, only a minority of those possessing a full house of morphological high-risk features progress to an ACS. The addition of shear and axial stress assessment and the quantification of trans-lesional gradients by computational fluid dynamics analysis appears to also provide additional information regarding risk of future rupture. ${ }^{12}$ It also may help predict plaque erosion, which comprises an increasing proportion of ACS, particularly in women. ${ }^{1314}$ 
FFR appears to have an association with these known plaque vulnerability features. ${ }^{15}$ Could it be that FFR provides a 'good enough' measure of vulnerability and lipid burden that avoids the excess intervention with an angiographic guided approach ${ }^{16}$ A pooled analysis of three randomised trials (with half of its patients drawn from the STEMI cohort of DANAMI-3-PRIMULTI), assessed the ability of the residual SYNTAX (Synergy between PCI with TAXUS and Cardiac Surgery) score after FFR guided 'complete' revascularisation to predict adverse events. ${ }^{17}$ Bearing in mind the limitations of post-hoc analyses and the mixed cohort, leaving angiographically significant lesions that were FFR negative did not appear to be associated with ischaemic outcomes after 2 years. It may be that physiological indices are a crude, but familiar and reproducible surrogate for abnormal plaque stress, but also that a significant component of a patient's risk may potentially be predicted by total coronary atheroma burden alone. Although it appears the functional characteristics of a lesion may describe some measure of its vulnerability, whether this relationship is associative, or causative, remains unknown.

\section{FUTURE DIRECTIONS}

Despite decades of advancement, vulnerable plaque identification still has several obstacles to overcome. First, a method of identifying plaques with a sufficient positive predictive value for adverse events is needed. We may find more success with the alternative approach of identifying the truly stable plaque where PCI could be safely deferred, or where novel and expensive therapies could be withheld.

Second, plaque assessment at baseline may be of limited value given that plaques can both deteriorate as well as stabilise over time on medical therapy. ${ }^{18}$ Invasive techniques or the use of ionising radiation are not ideal for serial assessment and magnetic resonance angiography may hold some promise in this area. ${ }^{19}$

Third, the identification of vulnerability on a lesion rather than patient level seems valuable only if an effective targeted local intervention is available. With the withdrawal of the absorb bioresorbable vascular scaffold (BVS) from the market, we are left with encouraging signs, ${ }^{20}$ but no conclusive evidence to support a strategy of PCI to vulnerable plaques. The PREVENT trial (NCT02316886) continues to recruit, randomising 1600 patients to drug eluting stent insertion versus optimal medical therapy for non-flow limiting disease with high-risk intracoronary imaging features, and OCT-CONTACT (NCT04878133) will randomise 460 patients to an OCT-guided strategy versus complete revascularisation. However, both studies are limited to only lesions with a $>50 \%$ angiographic stenosis. Whether contemporary drug eluting stents with ultrathin struts, the next generation of bioresorbable vascular scaffolds, or PCI to vulnerable plaque regardless of angiographic stenosis severity will be beneficial, remains unanswered.

\section{CONCLUSIONS}

FLOWER-MI is ultimately limited by its low event rate in evaluating an FFR versus an angiography-guided revascularisation strategy for non-culprit lesions after STEMI, and therefore, either a physiology or angiography guided approach still seem reasonable. However, trying to reduce adverse hard outcomes with physiology versus the blunt tool of angiography may only be a partial solution. Risk stratifying individual lesions with new and emerging techniques could allow a truly personalised approach to STEMI care. The pursuit of a clinically viable method to detect, and then intervene on the vulnerable plaque continues.

\section{Twitter Matthew E Li Kam Wa @mattlkw}

Contributors ML drafted the first manuscript. All authors (ML/KDS/CC/DP) contributed to the final manuscript. DP provided the original idea and is the guarantor of the paper.

Funding ML, KDS and DP are supported by grants from the British Heart Foundation (RE/18/2/34213), and the UK National Institute for Health Research through the Biomedical Research Centre award to King's College London and Guy's and St Thomas' NHS Foundation Trust.

Competing interests CC holds research grants from Biosensor, Coroventis Research, Medis Medical Imaging, Pie Medical Imaging, Cathworks, Boston Scientific, Siemens, HeartFlow and Abbott Vascular; and has received consultancy fees from HeartFlow, Opsens, Pie Medical Imaging, Abbott Vascular and Philips Volcano.

Patient consent for publication Not applicable.

Provenance and peer review Not commissioned; externally peer reviewed.

Open access This is an open access article distributed in accordance with the Creative Commons Attribution Non Commercial (CC BY-NC 4.0) license, which permits others to distribute, remix, adapt, build upon this work non-commercially, and license their derivative works on different terms, provided the original work is properly cited, appropriate credit is given, any changes made indicated, and the use is non-commercial. See: http://creativecommons.org/licenses/by-nc/4.0/.

ORCID iD

Matthew E Li Kam Wa http://orcid.org/0000-0002-0091-6022

\section{REFERENCES}

1 Thim T, van der Hoeven NW, Musto C, et al. Evaluation and management of Nonculprit lesions in STEMI. JACC Cardiovasc Interv 2020;13:1145-54.

2 Puymirat E, Cayla G, Simon T, et al. Multivessel PCI guided by FFR or angiography for myocardial infarction. $N$ Engl J Med 2021;385:297-308.

3 Tonino PAL, De Bruyne B, Pijls NHJ, et al. Fractional flow reserve versus angiography for guiding percutaneous coronary intervention. N Engl J Med 2009;360:213-24.

4 Mehta SR, Wood DA, Storey RF, et al. Complete revascularization with multivessel PCl for myocardial infarction. $N$ Engl $\mathrm{J} \mathrm{Med}$ 2019;381:1411-21

5 Tonino PAL, Fearon WF, De Bruyne B, et al. Angiographic versus functional severity of coronary artery stenoses in the fame study fractional flow reserve versus angiography in multivessel evaluation. J Am Coll Cardiol 2010;55:2816-21.

6 Madhavan MV, Kirtane AJ, Redfors B, et al. Stent-Related Adverse Events $>1$ Year After Percutaneous Coronary Intervention. J Am Coll Cardiol 2020;75:590-604.

7 Xaplanteris P, Fournier S, Pijls NHJ, et al. Five-year outcomes with PCl guided by fractional flow reserve. N Engl J Med 2018;379:250-9.

8 Ahmadi A, Leipsic J, Blankstein R, et al. Do plaques rapidly progress prior to myocardial infarction? the interplay between plaque vulnerability and progression. Circ Res 2015;117:99-104.

9 Piróth Z, Boxma-de Klerk BM, Omerovic E, et al. The Natural History of Nonculprit Lesions in STEMI: An FFR Substudy of the CompareAcute Trial. JACC Cardiovasc Interv 2020;13:954-61. 
10 Erlinge D, Maehara A, Ben-Yehuda O, et al. Identification of vulnerable plaques and patients by intracoronary near-infrared spectroscopy and ultrasound (PROSPECT II): a prospective natural history study. Lancet 2021;397:985-95

11 Pinilla-Echeverri N, Mehta SR, Wang J. Nonculprit lesion plaque morphology in patients with ST-Segment-Elevation myocardial infarction. Circulation 2020;13.

12 Lee JM, Choi G, Koo B-K, et al. Identification of High-Risk Plaques Destined to Cause Acute Coronary Syndrome Using Coronary Computed Tomographic Angiography and Computational Fluid Dynamics. JACC Cardiovasc Imaging 2019;12:1032-43.

13 Gijsen F, Katagiri Y, Barlis P, et al. Expert recommendations on the assessment of wall shear stress in human coronary arteries: existing methodologies, technical considerations, and clinical applications. Eur Heart J 2019;40:3421-33.

14 Dweck MR, Maurovich-Horvat P, Leiner T, et al. Contemporary rationale for non-invasive imaging of adverse coronary plaque features to identify the vulnerable patient: a Position Paper from the European Society of Cardiology Working Group on Atherosclerosis and Vascular Biology and the European Association of Cardiovascular Imaging. Eur Heart J Cardiovasc Imaging 2020;21:1177-83.
15 Lee JM, Choi KH, Koo B-K, et al. Prognostic implications of plaque characteristics and stenosis severity in patients with coronary artery disease. J Am Coll Cardiol 2019;73:2413-24.

16 Ahmadi A, Stone GW, Leipsic J, et al. Association of coronary stenosis and plaque morphology with fractional flow reserve and outcomes. JAMA Cardiol 2016;1:350-7.

17 Kobayashi Y, Lønborg J, Jong A, et al. Prognostic value of the residual SYNTAX score after functionally complete revascularization in ACS. J Am Coll Cardiol 2018;72:1321-9.

18 Räber L, Koskinas KC, Yamaji K, et al. Changes in coronary plaque composition in patients with acute myocardial infarction treated with high-intensity statin therapy (IBIS-4): a serial optical coherence tomography study. JACC Cardiovasc Imaging 2019;12:1518-28.

19 Hajhosseiny R, Bustin A, Munoz C, et al. Coronary magnetic resonance angiography: technical innovations leading us to the promised land? JACC Cardiovasc Imaging 2020;13:2653-72.

20 Stone GW, Maehara A, Ali ZA, et al. Percutaneous coronary intervention for vulnerable coronary atherosclerotic plaque. J Am Coll Cardiol 2020;76:2289-301. 